\title{
Young breast cancer patients in the developing world: incidence, choice of surgical treatment and genetic factors
}

\author{
Kruger WM, MBChB, BSc(Hons) in Reprod Medicine \\ General Practitioner with special interest in breast health in private practice \\ Apffelstaedt JP, MMed(Surg), FCS(SA), MBA \\ Professor in Surgery, University of Stellenbosch \\ Head: Mamma Clinic and Surgical Oncology Department, Tygerberg Hospital
}

Correspondence to: Prof JP Apffelstaedt, e-mail: justus@apffelstaedt.com

\section{Abstract}

Carcinoma of the breast is the most common cause of cancer in women in Western society. Although breast cancer occurs predominantly in older premenopausal and postmenopausal women, it also occurs in young women. Literature defines breast cancer in a young woman (or early onset breast cancer) as occurring in a woman less than 35 years of age. A diagnosis of breast cancer in a young woman impacts severely on all aspects of her life, as well as on those around her.

In Africa and other developing countries, the breast cancer burden is increasing and poor reporting and data availability may underestimate the exact numbers. The average age of diagnosis may be younger for women in developing countries than for women in developed countries. African patients are more likely to be premenopausal at diagnosis and the breast cancers tend to be more advanced at presentation than in other population groups in a country such as South Africa.

The choice of surgical treatment in early onset cancer depends on various factors. Young age is an independent risk factor for worse outcome regardless of whether a patient had a mastectomy or breast conserving therapy. Breast conserving treatment is an option for treatment of breast cancer in a young patient given the correct indications and that the patient is fully informed about the high risk of local recurrence.

The extent of genetic factors such as mutations on BRCA 1 and 2 (BReast CAncer 1 and 2) genes is still largely unknown on the continent of Africa, and much research still needs to be done. In the USA, only 5-10\% of early onset breast cancers are attributable to mutations on BRCA 1 and 2 genes, and another $15-20 \%$ of early onset breast cancers are due to gene polimorphisms and environmental factors.

General breast awareness among women of all age groups in Africa should be promoted. This includes how to perform self breast examinations and to seek urgent medical attention when a breast lump is discovered. In time, given the resources, good screening programmes on this continent to detect breast cancer at its earliest presentation would be the ideal.

SA Fam Pract 2007;49(9): 18-24

\section{Introduction}

In Western society and the world over, carcinoma of the breast is the most common cause of cancer in women. ${ }^{17}$ In South Africa, between 1993 and 1995 it has overtaken cervical cancer as the most common cancer in women. ${ }^{15}$ Breast cancer is mostly regarded as a disease occurring predominantly in older premenopausal and postmenopausal women. Much focus has been placed on these two groups of women over the years. However, breast cancer does occur in young women. In the United States, $2.7 \%$ of patients with breast cancer are younger than 35 years. ${ }^{13}$ It is reported that in the United States the incidence in women 20-24 years of age is 1.4 per 100,000 women, and for women aged 25-29 years 8.1 per 100,000 women. In women between the ages of 30-34 years, the incidence increases to 24.8 per 100,000 women. ${ }^{13}$

Although a relatively rare disease in young women, a diagnosis of breast cancer impacts severely on the young patient's life and future, extending to her family and society as a whole. It also impacts on the medical personnel involved in her treatment and care giving.

It has been shown that in young women, breast cancer has more aggressive biological features ${ }^{1,6}$ with more advanced disease at diagnosis ${ }^{13}$ and a poorer prognosis (especially in the $<30$ years age group) than older premenopausal patients. ${ }^{13}$ Much research still needs to be done on various aspects of breast cancer in this young population. Interesting topics arise, such as the contribution of BRCA 1 and 2 germ line mutations in this young population, as well as other genetic influences and factors. Another topic, mastectomy versus breast conservation therapy in treatment of the patient with early onset breast cancer, from the perspective of Africa as a developing continent, will be examined in this article through existing literature and available data.

\section{Breast cancer incidence rates in Africa as a developing continent.}

The breast cancer burden is increasing in developing countries. It is estimated that $70 \%$ of new cancer cases will occur in inhabitants of developing countries by 

the year 2020, and a vast number these cancers occurring in females will be of the breast. ${ }^{5,10}$ There are differences in the breast cancer incidence and mortality rates in the different geographical areas of the continent. This may be due to poor reporting and data availability, difficulty in estimating population denominators or different ethnic compounds from region to region. ${ }^{5}$ In developing countries, the average age at diagnosis of breast cancer may be younger than in developed countries, as data from eastern populations suggest.7.12,14

In eastern Africa, it is estimated that the age standardised incidence rates (per 100,000 women) is $19.5 . .^{9}$ In western Africa it is 27.8, in middle Africa 16.5 and 33.4 in southern Africa. ${ }^{9}$ Focusing on a country such as South Africa, the population consists of different ethnic groups. It is estimated that the lifetime risk for breast cancer in the white population of Anglo-European ancestry is $1 /$ 13 comparable with rates in other western countries. ${ }^{15}$ In women of mixed ethnicity there is a 1/63 lifetime risk of developing breast cancer, in Asian women (mainly of Indian origin) there is $1 / 21$ risk and in black South African women it is $1 / 81$ lifetime risk of developing breast cancer. ${ }^{15}$ Statistics are available through the National Cancer Registry, that reports cancers diagnosed by histology, cytology and haematology laboratories in South Africa.. ${ }^{2}$ The statistics may underestimate the cancer burden. ${ }^{15}$

It is estimated that the incidence is rising in the urban black African population. Walker AR et al found in a study that during the period 1994-1999, looking at hospital admissions of urban patients in Durban, the age adjusted annual incidence of breast cancer was 15.1/ 100,000 women, compared to previous figures of $5-10 / 100,000$ for the rural black population. ${ }^{16}$ Another study reported a doubling of breast cancer rates in women living in urban areas in the Western Cape compared with their rural neighbours. ${ }^{8}$ There are certain factors in the rural African population that lower the lifetime breast cancer risk. Late menarche, early age at birth of first child, multiparity and extended lactation all result in fewer ovulatory cycles, with lower endogenous oestrogen levels during a woman's lifetime. ${ }^{5,15,16}$ Other protective factors such as being physically active, a diet rich in vegetables and grains, and low alcohol consumption are cited as benefits of the rural lifestyle. 5,15,16 With urbanisation, the Western lifestyle is frequently adopted, and thus the differences in incidence rates of breast cancer.
Of importance is the fact that African patients are more likely to be premenopausal when diagnosed with breast cancer ${ }^{5}$ and present with breast cancer almost a decade earlier than their Western counterparts. ${ }^{17}$ Breast cancer incidence is highest between the ages of 35-45 years in western Africa. ${ }^{5}$

Breast cancer in the African population has also shown to be more advanced at presentation than in other population groups. Seventy to ninety per cent of African women of all age groups are diagnosed with breast cancer at stage III or IV. ${ }^{5}$ Vorobiof et al showed that $77.7 \%$ of stages 3 and 4 breast cancer were found in black women, compared to $30.7 \%$ in non black women, looking at medical records from several provincial hospitals from 1970$1997 .{ }^{15}$ This is somewhat explained by a delay in seeking medical attention. It is also postulated that the tumour biology may be inherently more aggressive. ${ }^{5}$

A need for breast health awareness from an early age is vital. An investigation done to establish the awareness of breast and cervical cancers among women of African descent in rural and urban South Africa, published in 2002, showed that one fifth of the respondents had not heard of these cancers and one third did not know about tests for breast cancer. Breast self examination techniques were only known by half of the population studied. ${ }^{11}$

\section{Mastectomy versus breast conserv- ing treatment in early onset breast cancer}

In a young patient with breast cancer, breast conserving treatment (BCT) is obviously a choice that is cosmetically desirable. In deciding which treatment option is best for a given patient, it is important to consider various factors. Young age is an independent risk factor for worse outcome in breast cancer regardless of whether the patient had BCT or a mastectomy. The young age risk factor is independent of clinical tumour size, clinical lymph node status, histological grade, hormone receptor status and systemic treatment. ${ }^{18}$

Young age ( $<35$ years) is a risk factor for local recurrence after BCT, compared to older patients who had BCT. Voogd et al found that women < 35years had a 9 times increased risk of local recurrence after BCT than women older than 60 years. Young patients who were treated with mastectomy had local recurrence rates similar to their older counterparts who were also treated with mastectomy. Other risk factors for local recurrence after BCT in all age groups were infiltrating tumour with an extensive intraductal component, vascular invasion and involvement of excision margins. ${ }^{13}$

Conservative surgery without radiotherapy was shown in various studies to have higher local recurrence rates in women with early onset cancer. ${ }^{18}$ In the EORTC (European Organisation for Research and Treatment fo Cancer) "boost versus no boost" randomised trial, local failure rate was reduced from $20 \%$ to $10 \%$ in patients < 40 years of age, a more pronounced result compared with patients $>50$ years

All patients with adjuvant systemic therapy, in the form of chemotherapy or hormonal therapy or both, have lower local recurrence rates after BCT. This applies to women of all age groups, although it is unknown to what degree local recurrence rates are reduced in women $<35$ years of age. ${ }^{18}$

The margin status of an excised tumour is regarded as an important factor in local failure in all age groups. Wide surgical excision and negative histological margins should reduce the local recurrence rate..$^{18}$ It has been found in the EORTC trial that young patients were found to have more DCIS (ductal carcinoma in situ), and that it was often of a higher grade. ${ }^{18}$

In a clinic based series of Robson et al of women with BRCA 1 and 2 who underwent breast conserving treatment, the risk of ipsilateral breast cancer was the same as for women without known mutations. But due to the lifetime risk of contralateral breast cancer and late ipsilateral breast cancer, bilateral mastectomy as prophylaxis should be a serious consideration. ${ }^{12}$

Breast conserving treatment need not be ruled out as a treatment option for young women in the developed or developing world, as long as the patient is fully aware of the high risk for local recurrence. There are no studies that show that overall survival is negatively impacted in patients receiving BCT. ${ }^{13}$

\section{Genetic factors and early onset can- cer}

In the USA, only $5-10 \%$ of early onset breast cancers are attributable to germ line mutations such as BRCA 1 on chromosome 17 and BRCA 2 on chromosome 13.4,6 Mutation rates are higher in patients with a strong family history ranging from $16-26 \%$ in BRCA 1 and $7-$ $13 \%$ in BRCA $2{ }^{4}$ In patients with BRCA 1 mutation, there is a $50 \%$ chance of developing breast cancer by age 50, and an $80 \%$ chance by 70 years of age. ${ }^{13}$ The lifetime risk for ovarian cancer in 


\section{Points to ponder:}

- Patients should be made aware of breast health from a young age.

- Encourage patients to go for annual mammograms from age 40 . If cost is a problem, at least then a mammogram every 2 years.

- If the woman's mother had breast cancer at age 40 , she should start screening ten years earlier. A specialised breast health centre can also do a genetic risk assessment for her.

A young woman with a palpable breast lump should be sent for further investigation:

\begin{tabular}{|l|l|}
\hline$\quad<$ age 25 & $\begin{array}{l}\text { Breast ultrasound plus an FNA (fine needle aspiration) of mass } \\
\text { if confirmed. }\end{array}$ \\
\hline - $\quad$ age 25 & $\begin{array}{l}\text { Breast ultrasound plus a mammogram if indicated and FNA/ } \\
\text { needle core biopsy of mass if confirmed. }\end{array}$ \\
\hline
\end{tabular}

these patients is 1 in 2. Breast cancer in BRCA 1 gene carriers is more likely grade 2 and 3 , oestrogen and progesterone receptor negative, and HER 2 negative. ${ }^{3}$ Also, $13 \%$ of these cancers are described as medullary or atypical medullary, compared to $1-3 \%$ of breast cancers described in routine practice. ${ }^{3}$ Breast cancers due to BRCA 2 gene mutation are less distinctive, but $80 \%$ of these tumours are oestrogen receptor positive. ${ }^{3}$ In African ancestry, breast cancer is less common, but it occurs at an earlier age and is more aggressive. Fackenthal et al analysed 39 young $\mathrm{Ni}$ gerian breast cancer patients and found that $74 \%$ carried at least one BRCA 1 and 2 variation with $69 \%$ having sequence variation in BRCA 2, representing a higher frequency of BRCA 2 variants compared to a previously studied white cohort. ${ }^{4}$ It is proposed that the significant genetic variation in BRCA 1 and 2 may contribute to breast cancer risk. No founder mutation was identified in this study. The extent of the contribution of BRCA 1 and 2 to the breast cancer burden in Africa is uncertain. ${ }^{5,15,4}$

Another $15-20 \%$ of breast cancers in young women are associated with gene polimorphisms and environmental factors, and the rest of the remaining breast cancer cases are thought to be sporadic. ${ }^{6}$ There are other rare genetic causes that predispose young women to breast cancer. Li- Fraumeni syndrome is a mutation of the TP53 gene on chromosome 17 . It is dominantly inherited, and causes paediatric soft tissue and bone sarcomas, leukaemia, brain, lung and adrenal cortical cancer, and breast cancer. ${ }^{13,6}$ Cowden syndrome is caused by a PTEN (phosphatase and tensin homoloque) gene mutation on chromosome 10, and the disease is associated with multiple hamartomas, and breast and thyroid cancer at a young age. ${ }^{13,6}$ Also, previous radiation during childhood and teenage years puts a young woman at higher risk for developing breast cancer.

\section{Conclusion}

Breast cancer does occur in young patients in Africa, a developing world. There is still much research to be done and accurate data collection needed on various aspects of early onset breast cancer in all regions of the continent. Breast conservation therapy is an option for treatment of breast cancer in the young patient given the correct indications. The extent of genetic factors such as mutations on BRCA 1 and 2 genes are still largely unknown on the continent of Africa. Hopefully, in the future, collaboration the world over will result in more knowledge of genetic factors, incidence and treatment of early onset breast cancer. General breast awareness among women of all age groups in Africa should be promoted, which includes breast-feeding and breast health: self breast examination must be taught and all women urged not to hesitate to visit a doctor if she discovers a breast lump, given that there are medical facilities close by. Screening programmes to detect breast cancer at its earliest presentation in women 40 years and older, from all socio-economic groups, would be an ideal. Unfortunately, on a continent where daily survival is a battle amidst violence, poverty and poor resources, hunger and AIDS, it is probably an unattainable dream for the moment.

\section{See CPD Questionnaire, page 42}

\section{PThis article has been peer reviewed}

\section{References}

1. Gonzalez-Angulo AM, Broglio K, Kau S et al Women age $<35$ years with primary breast carcinoma. Cancer 2005:103:2466-2472.

2. National Health Laboratory Service. Cancer Statistics 1997. (cited 2006 November). Available from: http://www.nh/s.ac.za

3. Eccles DM. Hereditary cancer: guidelines in clinical practice. Breast and ovarian genetics. Annals of Oncology 2004:15:133-38.

4. Fackenthal JD, Sveen L, Gao Q, Kohlmeir EK, Adebamowo C, Ogundiran TO, et al. Complete allelic analysis of BRCA1 and BRCA2 variants in young Nigerian breast cancer patients. J Med Genet 2005: 42:276281.
5. Fregene A, Newman LA. Breast cancer in sub-Saharan Africa: how does it relate to breast cancer in African American women? Cancer 2005;103:1540-1550.

6. Goldhirsch A, Gelber RD. Breast cancer in young women. In: Harris JR, Lippman ME, Morrow M, Osborne CK, editors. Diseases of the breast. Philadelphia: Lippencott Williams and Wilkins; 2004. p. 1339-1349.

7. Hisham AN, Yip CH. Spectrum of breast cancer in Malaysian women: Overview. World Journal of Surgery 2003;27:921-923.

8. Hoffman M, de Pinho H, Cooper D, Sayed R, Dent DM, Gudgeon A, et al. Breast cancer incidence and determinants of cancer stage in the Western Cape. SAMJ 2000;90:12121216.

9. International Agency for Research on Cancer. CANCERMondial Statistical Information System. Globocan database: summary table by cancer. (Cited 2007 June 11). Available from: http://www-dep.iarc.fr/

10. Jones SB. Cancer in the developing world: a call to action. BMJ 1999 Aug 21;319(7208): 505-508

11. Pillay AL. Rural and urban South African women's awareness of cancers of the breas and cervix. Ethn Health 2002 May; 7(2):103114

12. Robson M, Svahn T, McCormick B, Borgen $P$, Hudis CA, Norton L. Appropriateness of breast-conserving treatment of breast carcinoma in women with germline mutations in BRCA1 and BRCA2-a clinic based series. Cancer 2005; 103:44-51.

13. Shannon C, Smith IE. Breast cancer in adolescents and young women. Eur J Cancer 2003:39:2632-2642.

14. Son BH, Kwak BS, Kim JK, Kim HJ, Hong SJ, Lee JS, et al. Changing patterns in the clinical characteristics of Korean patients with breast cancer during the last 15 years. Archives of Surgery 2006;141:155-160.

15. Vorobiof DA, Sitas F, Vorobiof G. Breast cancer incidence in South Africa. J Clin Oncol 2001;19:125s-127s.

16. Walker AR, Adam FI, Walker BF. Breast cancer in black African women: a changing situation. J R Soc Health 2004;124: 81-85.

17. Xiong Q, Valero V, Kau V, Kau SW, Taylor S, Smith TL, et al. Female patients with breast carcinoma age 30 years and younger have a poor prognosis: the MD Anderson Cancer Center experience. Cancer 2001;92:2523 2528.

18. Zhou P, Recht A. Young age and outcome for women with early-stage invasive breast carcinoma. Cancer 2004;101:1264-1274.

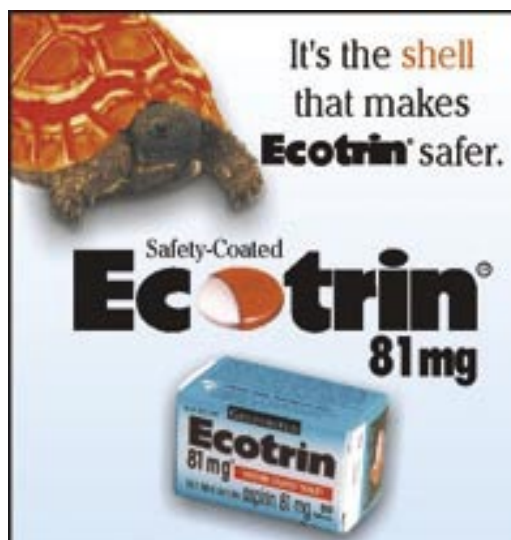

The miracle of Aspirin made safer.

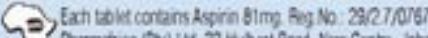

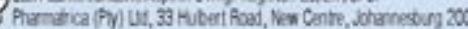

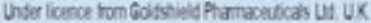




\section{Case study 1:}

A 34 year old female patient presented with a palpable lump in her right breast first felt a month previously. Clinically the mass was at $8 \mathrm{h00}, 2 \mathrm{~cm}$ from the nipple in the right breast, and it felt firm, mobile, $2 \times 2 \mathrm{~cm}$ in diameter. Mammogram showed pleomorphic calcifications right inferolateral in the area of the mass (Figures 1, 2 and 3). Ultrasound showed an oval hypoechoic mass, poorly circumscribed (Figure 4). FNA (fine needle aspiration) was done under ultrasound guidance and cytology was highly suspicious of malignancy. A needle core biopsy was done under ultrasound guidance to confirm the diagnosis and histology was compatible with invasive ductal carcinoma.

Figure 1: Full field digital mammogram CC (cranio-caudal) view right breast: a group of pleomorphic micro calcifications noted inferolateral in area of palpable mass.

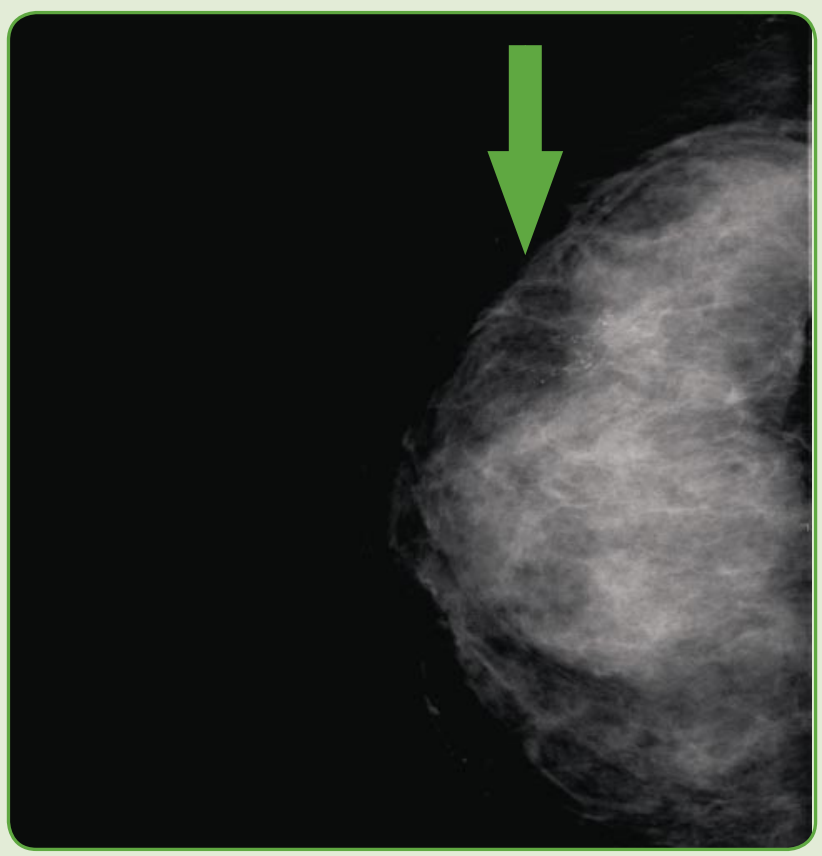

Figure 3: Full field digital mammogram spot magnification CC (cranio-caudal) view of the right breast: the group of pleomorphic micro calcifications inferolateral clearly visible.

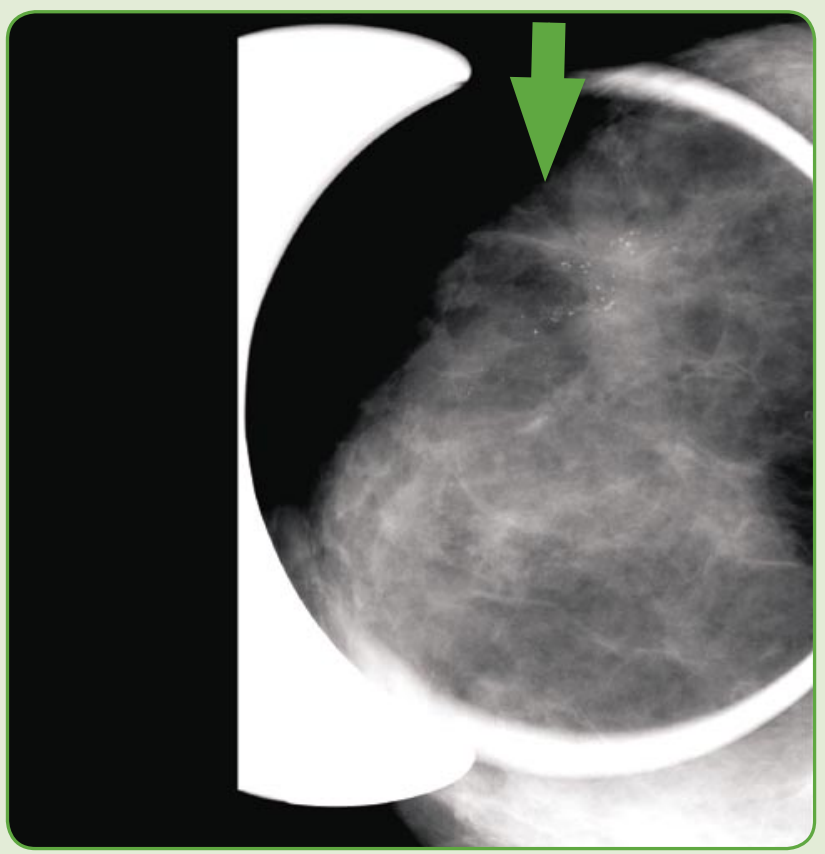

Figure 2: Full field digital mammogram MLO (mediolateral oblique) view of the right breast: a group of pleomorphic micro calcifications inferolateral in the area of the palpable mass.

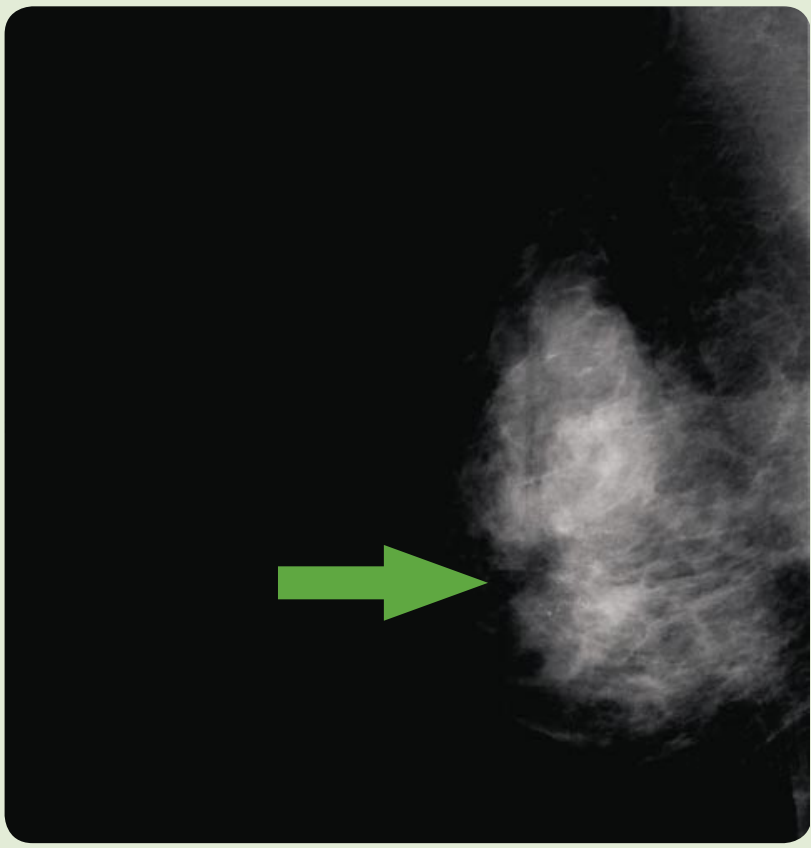

Figure 4: Ultrasound image of the lesion in the right breast: oval nonhomogeneous mass with mixed hypo- and hyperechogenicity and ill defined margins. Histology compatible with invasive ductal carcinoma.

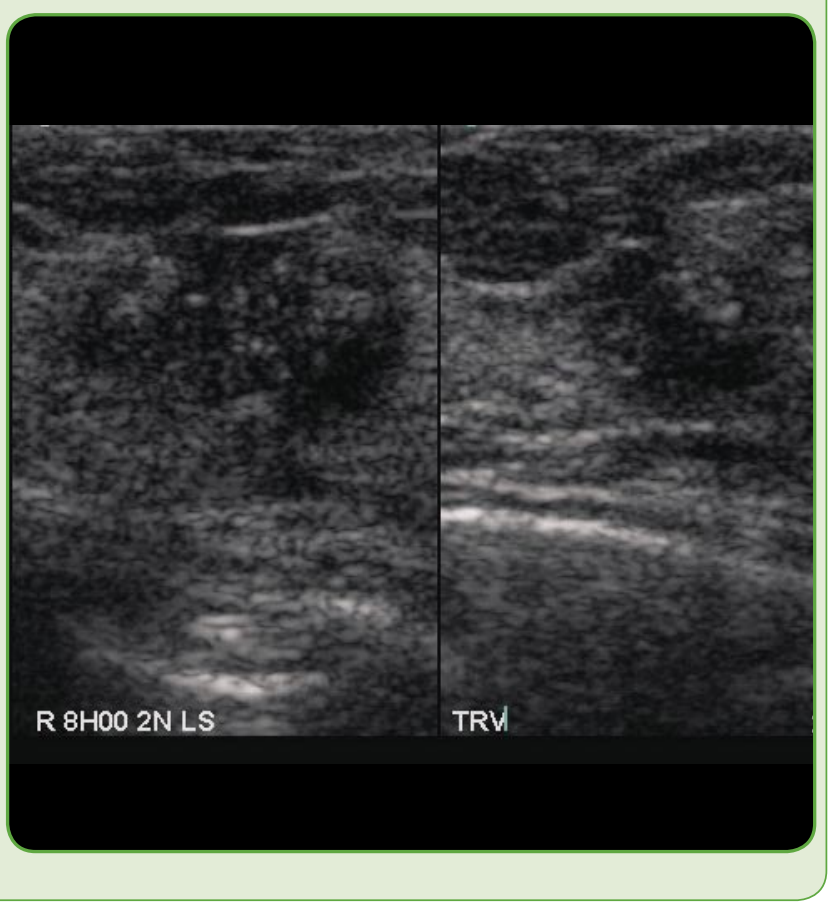




\section{Case Study 2:}

A 30 year old female patient presented with a mass in the left breast present for the past 5 months. On examination the mass was palpable, $2 \times 2 \mathrm{~cm}$ in the left breast at 5 h00. Mammogram showed a spiculated lesion inferolateral left breast. See Figures 5 and 6. Ultrasound showed a nonhomogeneous hypoechoic lesion with poorly defined margins and posterior acoustic shadowing. See Figure 7. FNA (fine needle aspiration) was done under ultrasound guidance and cytology showed hyperplastic ductal epithelial cells, but the lesion looked suspicious on mammogram and ultrasound, and a needlecore biopsy or excision biopsy had to be done. The patient chose an excision biopsy, and histology confirmed classic invasive lobular carcinoma.

Figure 5: Full field digital mammogram MLO (mediolateral oblique) view of the left breast: spiculated mass inferolateral.

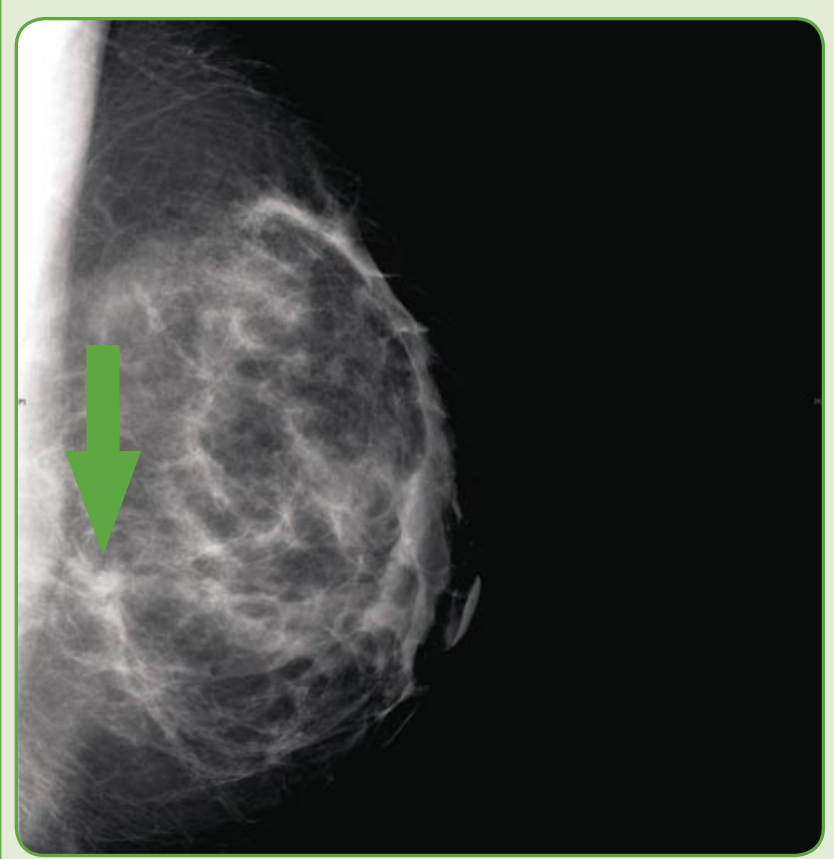

Figure 6: Full field digital mammogram CC (cranio-caudal) view of the left breast: spiculated mass infero-lateral.

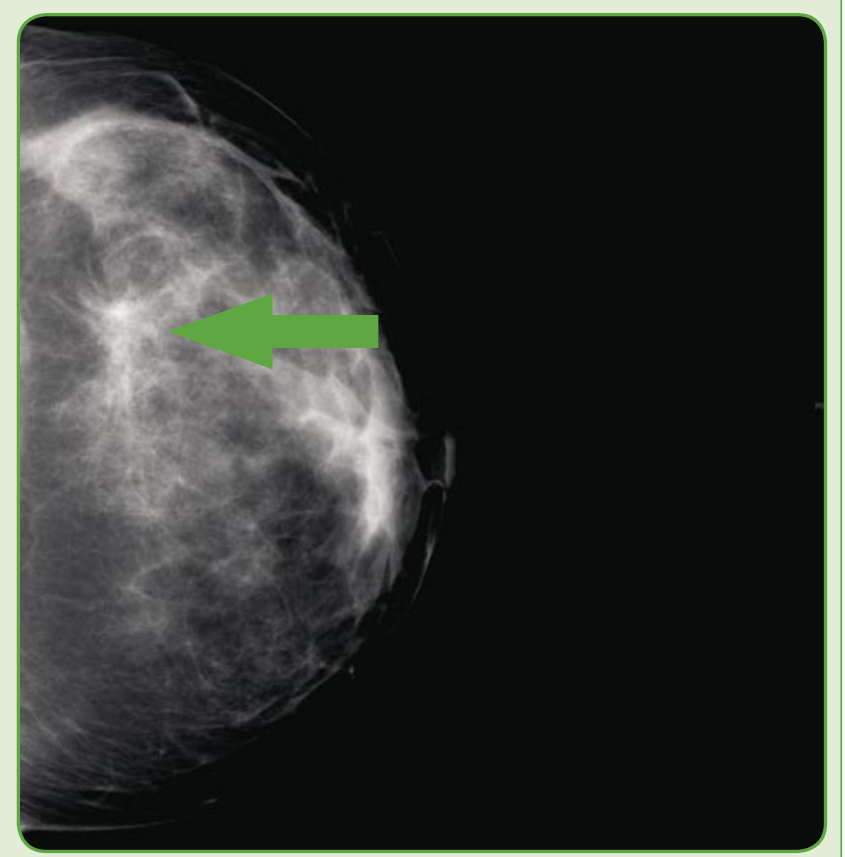

Figure 7: Ultrasound image of the palpable lesion in the left breast: nonhomogeneous hypoechoic mass with irregular contours, ill defined margins and posterior acoustic shadowing. Histology compatible with invasive lobular carcinoma.

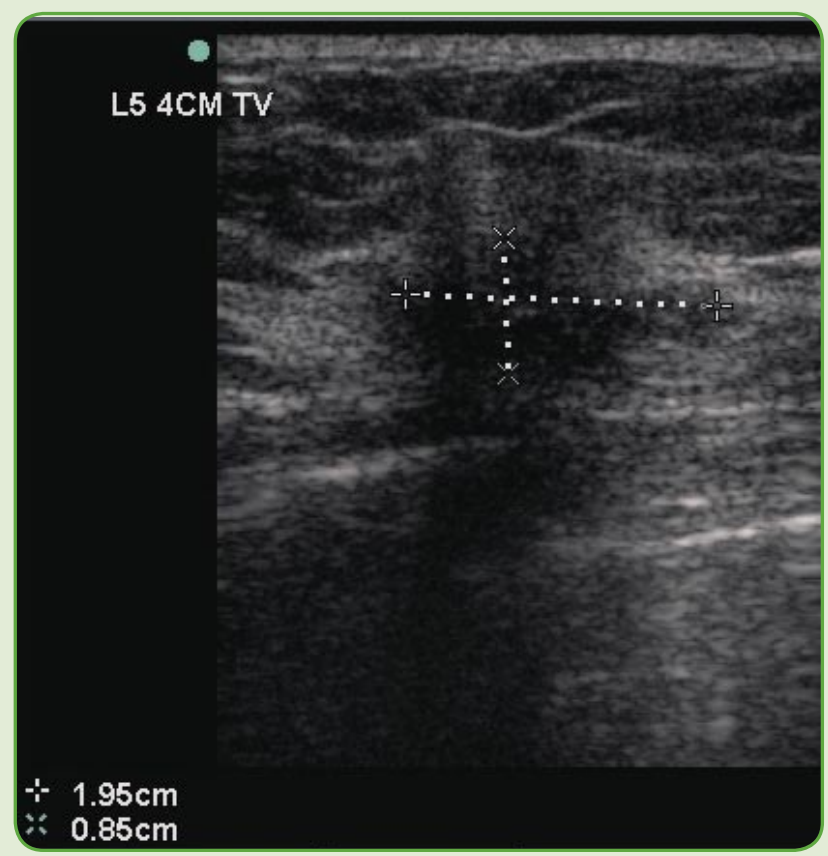

